Session:

\title{
Experimental Visualizations of a Generic Launch Vehicle Flow Field: Time-Resolved Shadowgraph and Infrared Imaging
}

\author{
Theodore J. Garbeff $\mathrm{II}^{*}$, Jayanta Panda ${ }^{\dagger}$, James C. Ross* \\ NASA Ames Research Center, Moffett Field, Ca, 94035
}

Time-Resolved shadowgraph and infrared (IR) imaging were performed to investigate off-body and onbody flow features of a generic, "hammer-head" launch vehicle geometry previously tested by Coe and Nute (1962). The measurements discussed here were one part of a large range of wind tunnel test techniques that included steady-state pressure sensitive paint (PSP), dynamic PSP, unsteady surface pressures, and unsteady force measurements. Image data was captured over a Mach number range of $0.6 \leq M \leq 1.2$ at a Reynolds number of 3 million per foot. Both shadowgraph and IR imagery were captured in conjunction with unsteady pressures and forces and correlated with IRIG-B timing. High-speed shadowgraph imagery was used to identify wake structure and reattachment behind the payload fairing of the vehicle. Various data processing strategies were employed and ultimately these results correlated well with the location and magnitude of unsteady surface pressure measurements. Two research grade IR cameras were positioned to image boundary layer transition at the vehicle nose and flow reattachment behind the payload fairing. The poor emissivity of the model surface treatment (fast PSP) proved to be challenging for the infrared measurement. Reference image subtraction and contrast limited adaptive histogram equalization (CLAHE) were used to analyze this dataset. Ultimately turbulent boundary layer transition was observed and located forward of the trip dot line at the model sphere-cone junction. Flow reattachment location was identified behind the payload fairing in both steady and unsteady thermal data. As demonstrated in this effort, recent advances in high-speed and thermal imaging technology have modernized classical techniques providing a new viewpoint for the modern researcher.

\section{Introduction}

$\mathrm{T}_{\mathrm{i}}$ ime-Resolved shadowgraph and infrared (IR) imaging were performed to investigate off-body and on-body flow features of a generic, "hammer-head" launch vehicle geometry previously tested by Coe and Nute ${ }^{1}$. The purpose of this wind tunnel test was to deploy state of the art instrumentation on a well understood model to advance future test techniques, and provide a large database for the validation of Computational Fluid Dynamics (CFD) codes. The measurements discussed here were one part of a large range of wind tunnel test techniques that included steadystate pressure sensitive paint (PSP), dynamic PSP, unsteady surface pressures, and unsteady force measurements. The

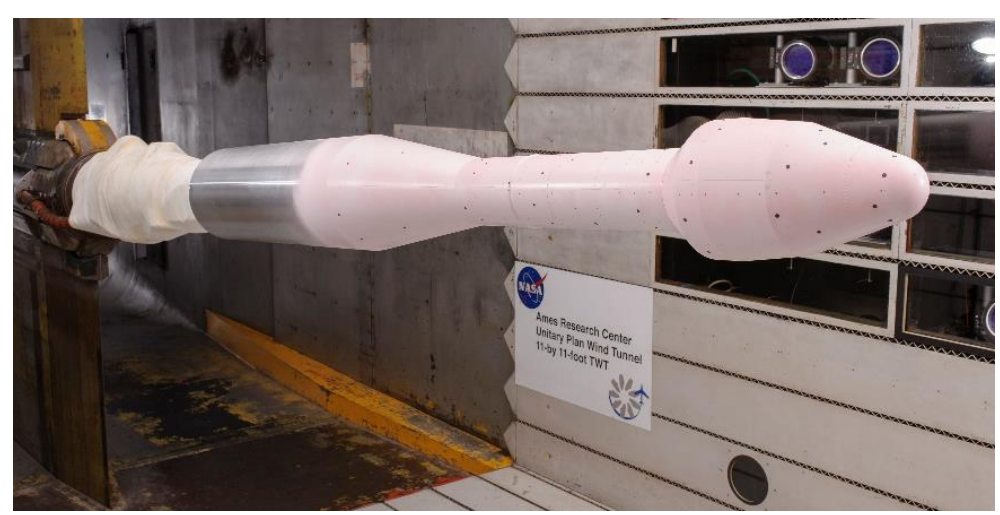

Figure 1: Generic Launch Vehicle, Ames UPWT 11 by 11 foot test was conducted in the 11-by-11 foot test section of the Unitary Plan wind tunnel at NASA Ames Research Center (Figure 1).

At the NASA Ames UPWT the schlieren/ shadowgraph system is a classic Z-type setup consisting of a high-powered light emitting diode light source, two light collimating four-foot diameter mirrors, optical quality test section glass, digital cameras with associated optics and data systems ${ }^{2,3}$. Shadowgraphy is done for the majority of testing at the Ames UPWT as it is more sensitive to the gradients produced by shock waves and fine scale turbulence ${ }^{4}$. For this

\footnotetext{
* Aerospace Engineer, Wind Tunnel Systems Branch, MS 227, Member AIAA

${ }^{\dagger}$ Aerospace Engineer, Experimental Fluid Mechanics Branch, MS 227, Member AIAA

* Aerospace Engineer, Experimental Fluid Mechanics Branch, MS 227, Member AIAA
}

American Institute of Aeronautics and Astronautics 
application, a single Phantom v2010 high-speed camera was used to capture shadowgraph imagery of wake structure and reattachment behind the payload fairing of the vehicle. Typical acquisition parameters were 40,000 frames per second (FPS), six microsecond exposure times, with 5,300 frames collected at each point. Shadowgraph Image data was captured over a Mach

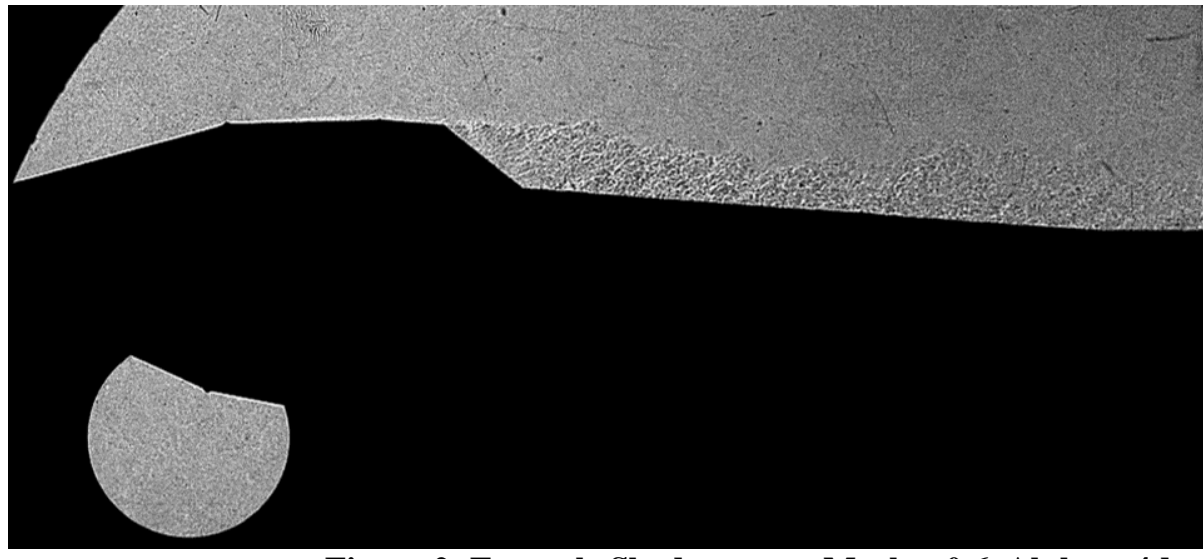

Figure 2: Example Shadowgram, Mach = 0.6, Alpha = 4deg number range of $0.6 \leq \mathrm{M} \leq 1.2$ at a Reynolds number of 3 million per foot. Figure 2 details an example still image taken from a high-speed image burst at subsonic conditions. Optical access at the 11 by $11 \mathrm{Ft}$ test section is limited by the rectangular window frames and slotted wall baffles (see Figure 1). Additionally, as this test also served as a demonstration for dynamic pressure sensitive paint and infrared flow visualization, the center window frame was occupied by cameras save for a circular through path. Therefore, only the upper half of the model was afforded an unobstructed light path during a small portion of the wind tunnel test. While no shock features are present in Figure 2 (subsonic regime) the separated flow is clearly visible behind the payload fairing, demonstrating the sensitivity of the system.

In order to visualize structures in the flow field, it proved advantageous to compute statistics at each pixel location across time throughout the entire image field. Figure 3 demonstrates contours of pixel standard deviation magnitudes computed from the high-speed shadowgraph image data. The upper image was computed from data at Mach $=0.6$. The flow separation behind the fairing, wake structure and subsequent reattachment is clearly visible as

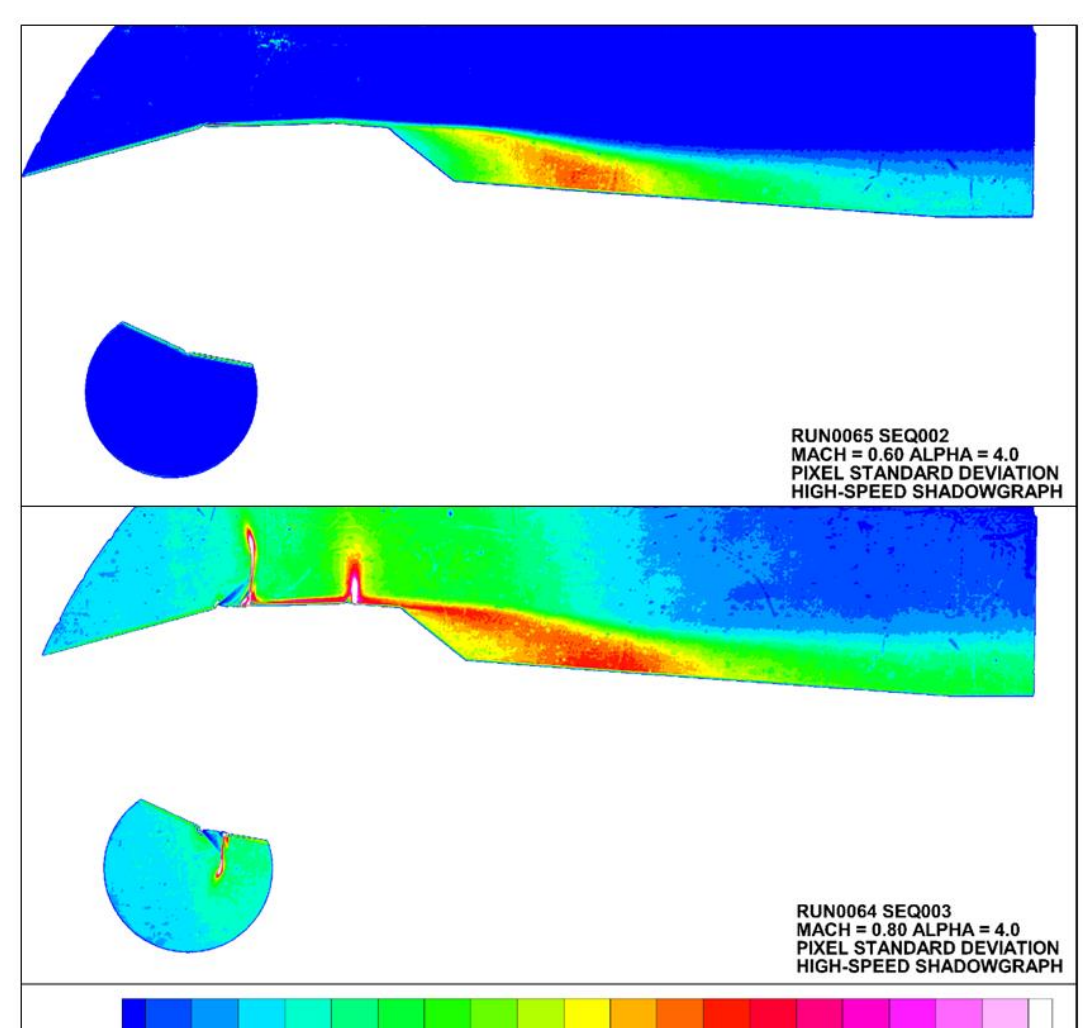

Figure 3: Shadowgraph Pixel Intensity Standard Deviation high values in the standard deviation contours. The lower portion of Figure 3 shows contours of standard deviation but at the onset of transonic flow. Local expansions with subsequent shocks can be identified around the upper portion of the fairing. The size and magnitude of fluctuating wake behind the payload fairing is also evident. Additionally, the background contains higher contour values marking an overall increase in freestream turbulence intensity at the higher Mach number. These results were compared with the unsteady surface pressure measurements taken congruently with the image data. Figure 4 qualitatively compares the magnitude and location of the unsteady surface pressures with the contours of pixel intensity statistics previously discussed. The magnitude of surface pressure variation has been mapped onto a three dimensional model of the test article ${ }^{5}$. In both instances the area of wake reattachment compares well between 
the two measurement methods. At Mach 0.8 the local shocks recorded in the shadowgraph data are not clearly captured in the surface pressure measurements. This is likely due to the comparatively coarse resolution of the discrete surface pressure sensor locations. These results help to provide a complete picture of the experiment by merging onbody and off-body measurements.

In order to extract instantaneous structure from the high-speed shadowgraph image data, a spatial filtering scheme was employed. Convolving an image instance with a variance computation identified regions of high spatial structure variation. Next, an image threshold operation was performed to binarize a particular region of interest.
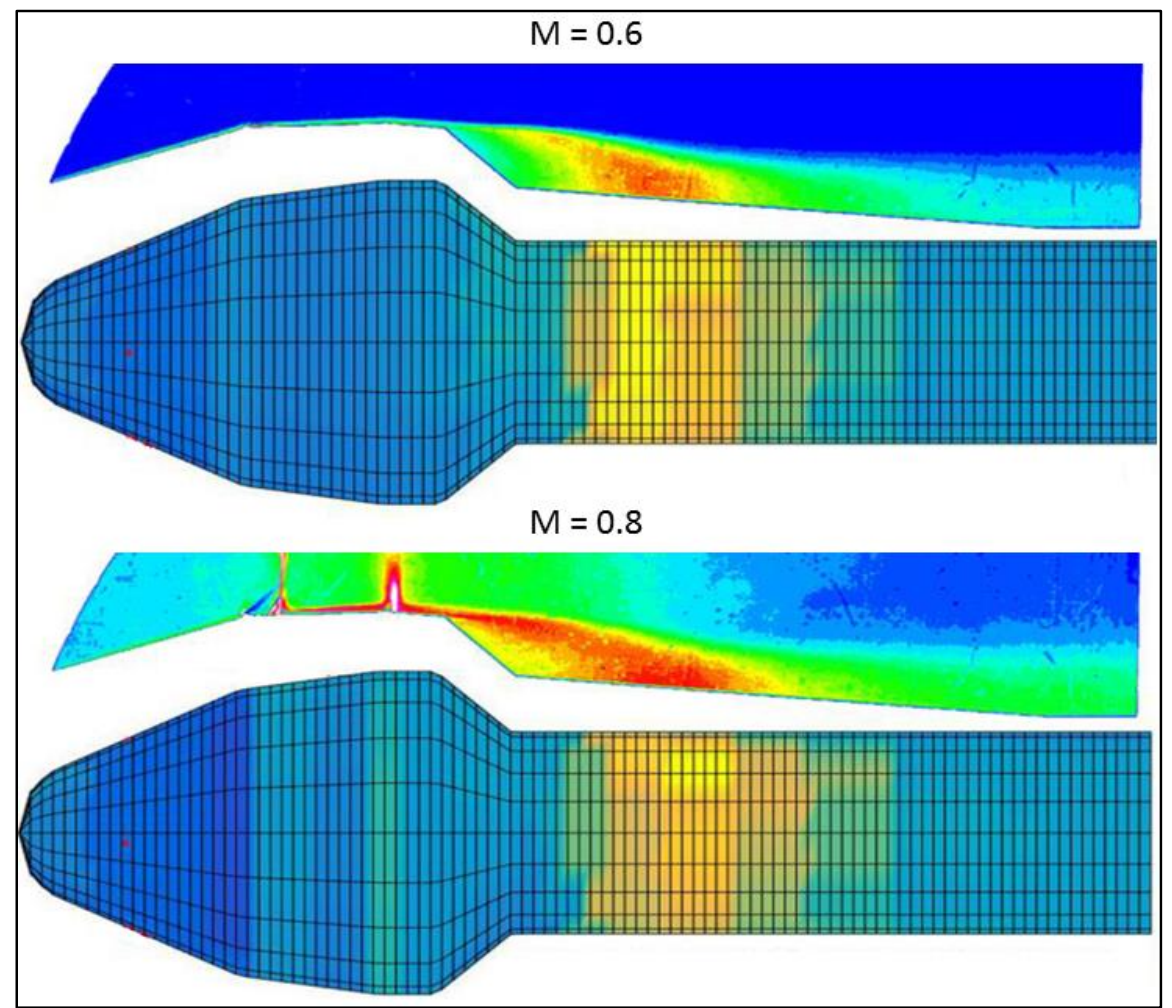

Figure 4: Shadowgraph Versus Surface CpRMS Finally, an edge detection routine was used to determine contours and subsequently extract contour geometry. Figure 5 depicts this process in order to extract the wake curvature. The most upper image depicts a time instance that has been filtered, thresholded, and binarized to extract the wake geometry. Edge detection was then performed and a contour fitted (green line overlay). Finally, the wake contour curvature is computed and plotted with respect to tunnel $\mathrm{x}$-coordinate. As the wake structure breaks down and convects downstream, its curvature increases.

Power spectral densities were computed at various regions of the flow field. At a particular location a contextual region was placed and the mean pixel intensity was computed across the image set, creating an averaged

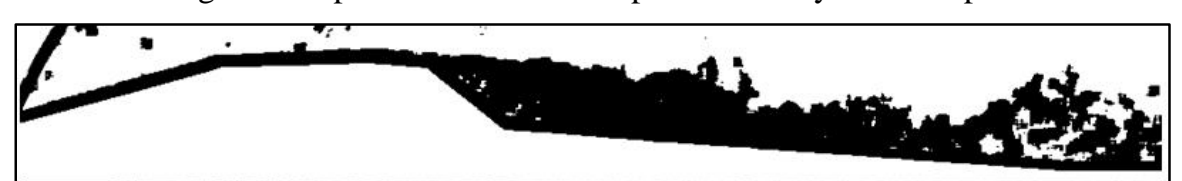
pixel intensity time history. Given these time histories the data was windowed and power spectral densities were

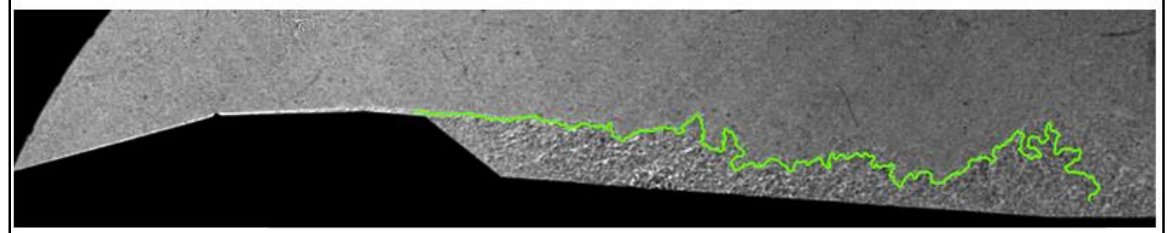
computed. Figure 6 depicts several example spectra computed at different regions in the flow field at Mach $=1.20$ and alpha $=0$. Probes were taken at a "free-stream" location, within the wake of the

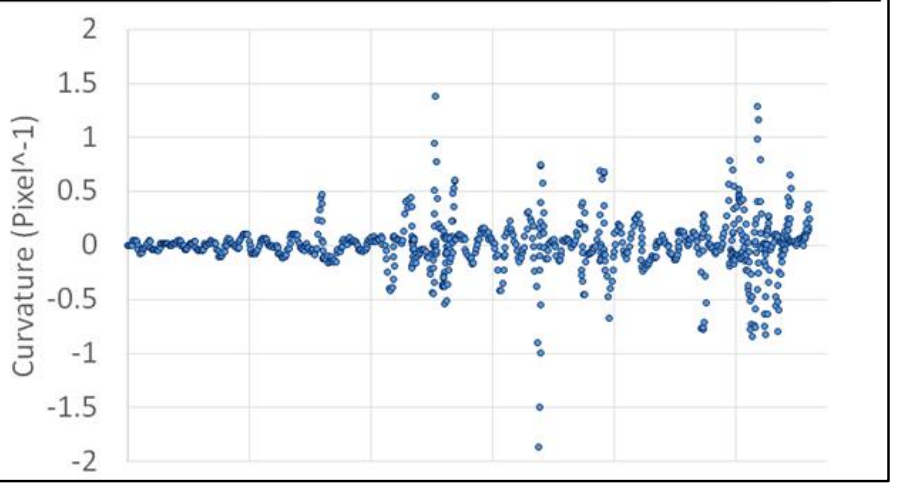
fairing, and near the spherecone junction. The free-stream probe revealed spectra that were strongly dominated by background tunnel tones occurring at approximately $2800 \mathrm{~Hz}$, a value corroborated by unsteady surface pressure measurements. The sphere-cone junction region was examined as it showed large magnitudes

Figure 5: Wake Geometry Extraction in $\mathrm{Cp}$ RMS as indicated by the

American Institute of Aeronautics and Astronautics 
unsteady pressure measurements. Additionally, the shadowgraph data contained Mach waves emanating from this region. The spectra at this location were strongly influenced by the background tunnel tones, indicating that this feature might be driven by these tunnel tones. The wake region also showed some influence of the tunnel tones. However, this could be an artifact of the measurement technique as the entire flow field is integrated, possibly contaminating a particular region of interest. As these background tunnel tones seem to have a particular geometry and orientation it should be possible to filter

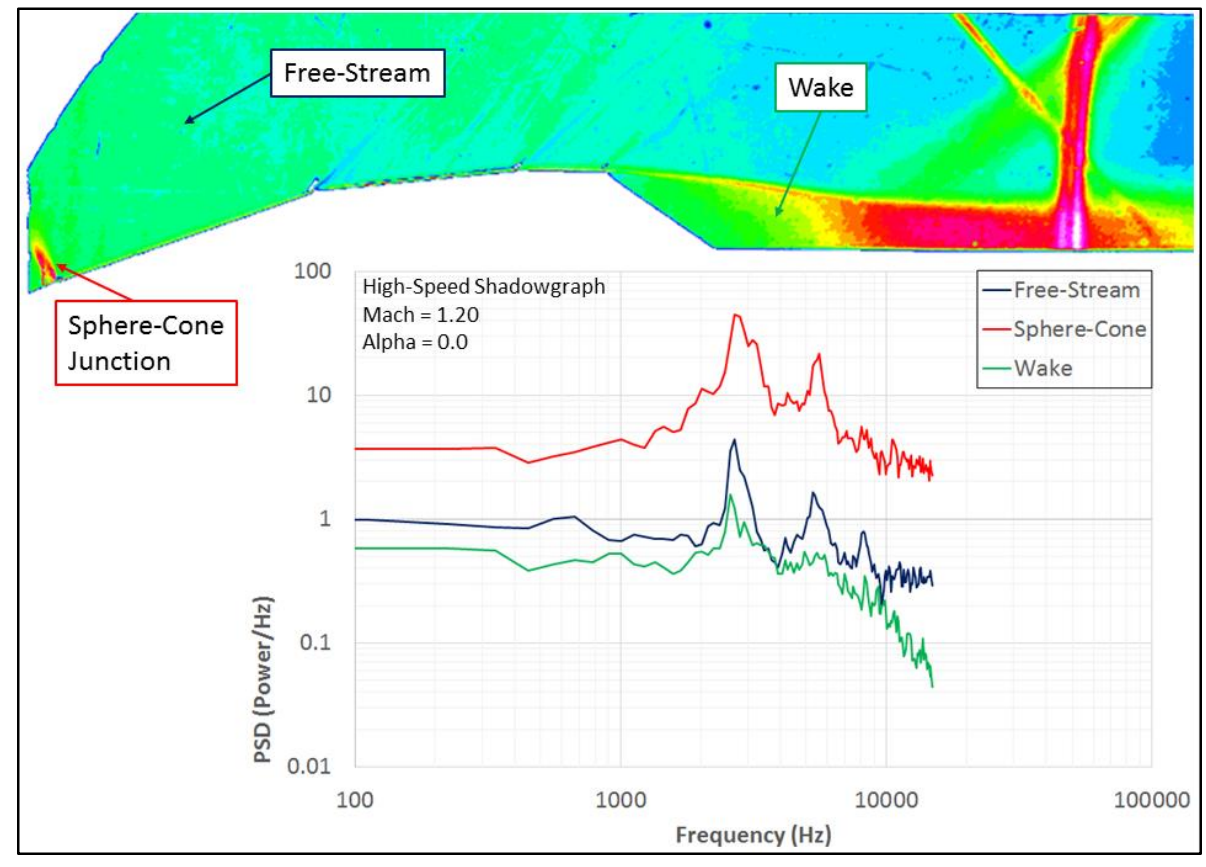

Figure 6: Power Spectral Densities these features using gradient base image convolution operations.

Two FLIR mid-wave (3-5 um), research grade infrared cameras were positioned to image boundary layer transition at the vehicle nose and flow reattachment behind the payload fairing. The IR camera imaging flow reattachment was run at high frame rates (400 FPS) to capture unsteady phenomena. The poor emissivity of the model surface treatment (fast PSP) proved to be challenging for the infrared measurement. Reference image subtraction and contrast limited adaptive histogram equalization ${ }^{6}$ were used to analyze this dataset. Figure 7 depicts turbulent boundary layer transition on the nose of the model. This image was rendered by subtracting a wind-off reference image that was adaptively scaled to match the background average pixel intensity. This approximated for differences in tunnel background temperature and consequently the magnitude of reflection to be subtracted. Ultimately turbulent boundary layer transition was observed and located forward of the trip dot line neat the model sphere-cone junction. The high frame rate IR camera imaging the portion of the model aft of the payload fairing was able to identify the

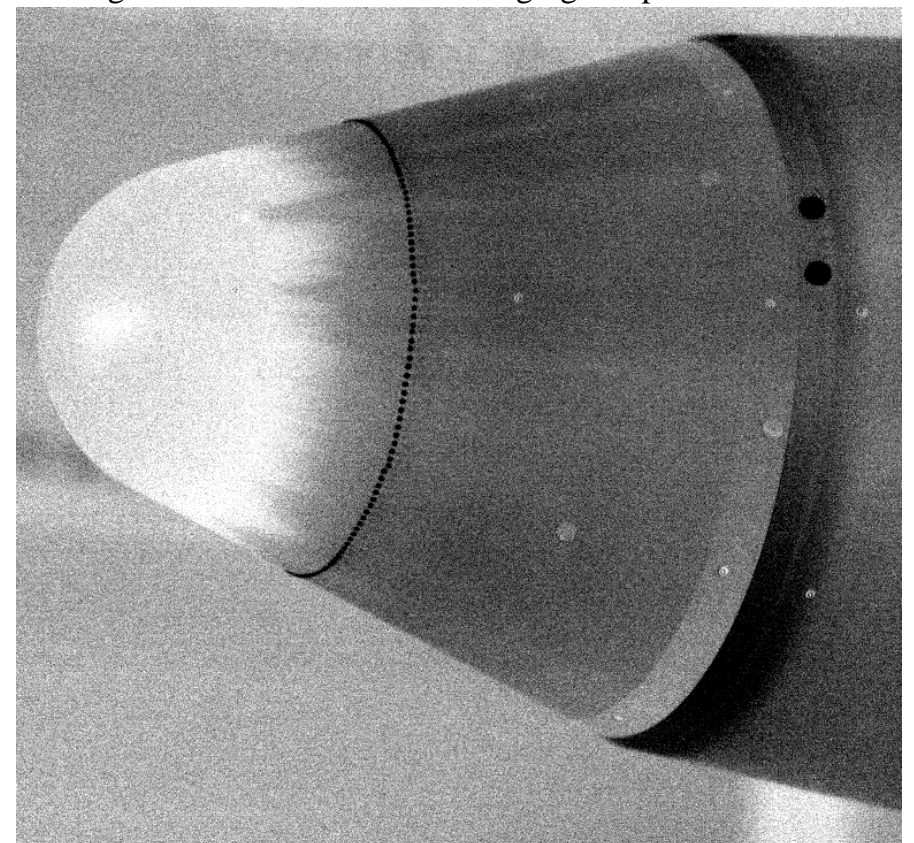

Figure 7: Boundary Layer Transition region of flow reattachment. This involved a sequential image averaging and subtraction technique. The pixel average was computed across a subset of images and each averaged image was subtracted from the next. Finally the variance across the set of subtracted images was computed to highlight flow features changing with time. Figure 8 depicts an example set of data processed using this strategy. The gradient from low to high pixel intensity in the region aft of the payload fairing indicated an increase in the magnitude of the surface temperature fluctuation due to flow reattachment.

As demonstrated in this effort, recent advances in high-speed and thermal imaging technology have allowed for a new capability to both quantify and qualify features of a flow field that has before been difficult or impossible to obtain. By integrating these imaging systems within the larger wind tunnel instrumentation suite, data can be collected with no impact on test productivity and synchronized across all measurement types.

American Institute of Aeronautics and Astronautics 
Advancements in image processing and computing hardware has allowed for processed data products made available to the researcher real-time. Some of these data products provide context to existing measurement techniques (CpRMS compared with shadowgraph pixel statistics, flow reattachment location compared with pressure data, shadowgraph pixel spectra compared with acoustic measurements). Some of these optical data products replace other techniques that are too costly (IR

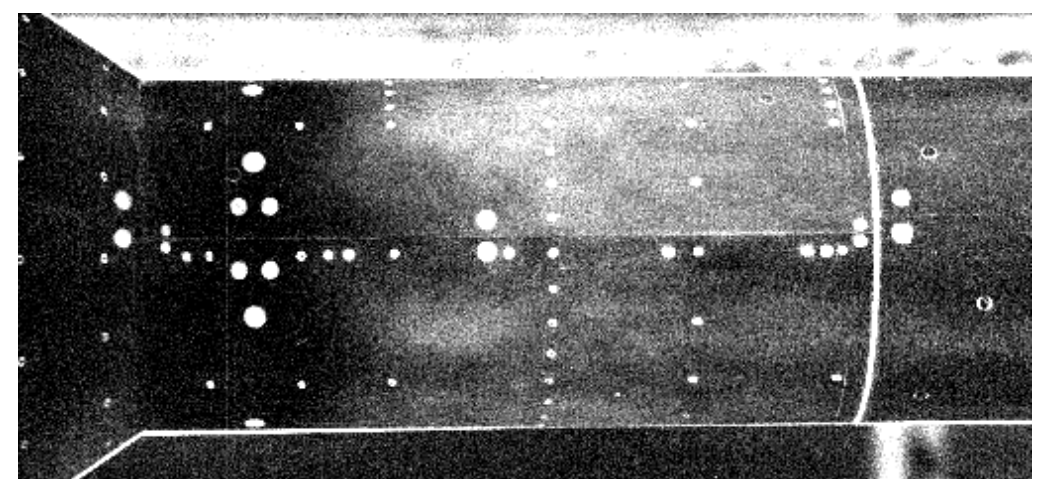

Figure 8: High Frame Rate IR, Wake Reattachment, Mach = 1.10

boundary layer transition detection versus sublimation). Finally, a few of these data products likely inform a unique capability (time-resolved wake geometry extraction, image filtering based on flow feature geometry). Ultimately these test techniques produce large volumes of information rich data providing the modern researcher with powerful tools to help guide the design of the next generation of flight vehicles.

\section{References}

${ }^{1}$ C. F. Coe, J.B. Nute: Steady and Fluctuating Pressures at Transonic Speeds on Hammerhead Launch Vehicles. NASA Technical Memorandum X-778, 1962.

${ }^{2}$ T.J. Garbeff II, J.T. Heineck, et al: Institutional Schlieren: A Production-Level Wind Tunnel Test Measurement. 53rd AIAA Aerospace Sciences Meeting, AIAA SciTech.

${ }^{3}$ G. Lee: Study of Optical Techniques for the Ames Unitary Plan Wind Tunnels Pat 1. schlieren. NASA-CR-189951, 1992

${ }^{4}$ G.S. Settles: Schlieren and Shadowgraph Techniques. Springer-Verlag, New York, 2001

5 J. Panda, et al: Unsteady Pressure Fluctuations Measured on a Generic Space Vehicle and Comparison with Coe \& Nute's (1962) Data. 55th AIAA Aerospace Sciences Meeting, AIAA SciTech.

${ }^{6}$ S. M. Pizer, E. P. Amburn, J. D. Austin, et al.: Adaptive Histogram Equalization and Its Variations. Computer Vision, Graphics, and Image Processing 39 (1987) 355-368.

American Institute of Aeronautics and Astronautics 